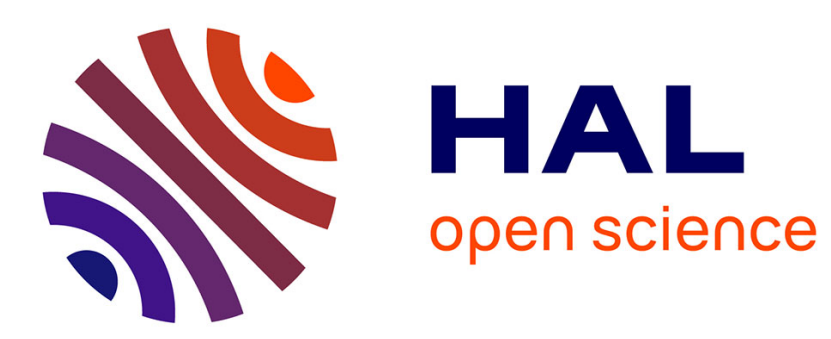

\title{
Revisiting Backoff algorithms in CSMA/CA based MAC for channel Reservation in RFID reader Networks through broadcasting
}

\author{
Ibrahim Amadou, Nathalie Mitton
}

\section{- To cite this version:}

Ibrahim Amadou, Nathalie Mitton. Revisiting Backoff algorithms in CSMA/CA based MAC for channel Reservation in RFID reader Networks through broadcasting. Wimob -9th International Conference on Wireless and Mobile Computing, Networking and Communications - 2013, Oct 2013, Lyon, France. hal-00850240

\author{
HAL Id: hal-00850240 \\ https://hal.inria.fr/hal-00850240
}

Submitted on 5 Aug 2013

HAL is a multi-disciplinary open access archive for the deposit and dissemination of scientific research documents, whether they are published or not. The documents may come from teaching and research institutions in France or abroad, or from public or private research centers.
L'archive ouverte pluridisciplinaire HAL, est destinée au dépôt et à la diffusion de documents scientifiques de niveau recherche, publiés ou non, émanant des établissements d'enseignement et de recherche français ou étrangers, des laboratoires publics ou privés. 


\title{
Revisiting Backoff algorithms in CSMA/CA based MAC for channel Reservation in RFID reader Networks through broadcasting
}

\author{
Ibrahim Amadou, Nathalie Mitton \\ Inria Lille - Nord Europe - France, Email: firstname.lastname@inria.fr
}

\begin{abstract}
Mitigating reader-to-reader collisions is one of the principal challenges in a large-scale dynamic RFID system with a number of readers deployed in order to maximize the system performance (i.e., throughput, fairness and latency). In prior works, contention-based and activity scheduling medium access control (MAC) protocols are commonly used approaches to reduce such problems. Existing protocols typically perform worse in a large-scale RFID dynamic system and require more additional components or are based on unrealistic assumptions. So far, many research efforts have been made to improve the performance or the reliability of Carrier Sense Multiple Access (CSMA) techniques for Mobile Ad-Hoc Networks (MANETs) by using an adaptive Backoff schemes. In this paper, we look at these well known solutions that proved their efficiency in high congestion wireless networks. We evaluate the performance and characterize these solutions when they are used to reserve the wireless channel through broadcasting message for reader-to-tag communication. Based on the application requirements, we study their capacity to mitigate collisions, the channel access latency, the average number of successful requests sent per reader and the fairness index in the context of RFID networks.
\end{abstract}

\section{INTRODUCTION}

Radio Frequency IDentification (RFID) systems [6] is a short-range communication technology that have generated considerable attention recently due to many applications such as supply markets, localization and objects tracking, activity monitoring, and access control and security. An RFID system consists of Radio Frequency (RF) tags and RFID readers. From application viewpoint, RFID tags are classified in two families: passive and active. Passive tags have no battery, and they acquire their power supply from the radio communication wave of the reader. It also means that tag-to-reader communication is highly constrained by the received power at tags. Instead active tags have their own power supply. While, RFID readers have more complex design with less constraints and high radio communication range. The reader radio communication range could be around of several meters based on the operational radio frequency specification and the transmission power. In various applications of RFID systems, the mostly widely used RFID tags are passive. Reader usually initiates communication with the tags through request/response process. It acts as a power supplier and operator of the passive tags. They are primarily designed to identify objects with unique identifiers in a contactless fashion. Therefore, misreading RFID tag is not allowed in such systems. To avoid this problem in large-scale deployment applications of RFID systems, RFID readers are generally deployed in large number allowing the coverage of the interested environment.

However, due to readers close proximity, when nearby readers simultaneously try to communicate with tags located within their interrogation range, serious interference problems may occur. This is mainly due to the overlapping of readers' field. Such interferences may cause signal collisions that lead to the reading throughput barrier and degrade the system performance. Therefore, the design of an efficient readerto-reader anti-collision protocol has emerged as the most interesting research issues in recent years. The key to make the RFID system efficient is to schedule readers activities so that neither interferences nor collisions may occur.

These protocols can be broadly classified as CSMA-based and activity scheduling based through time division (TDMA), frequency division (FDMA) or by putting together both approaches. The former approach is considered as an efficient approach (i.e. Fairness) in large-scale RFID readers networks because it is full-distributed algorithm. Like carrier sense multiple access with collision avoidance technique it does neither need synchronization nor additional resource (e.g. server) like in the latter approach. Nevertheless, existing CSMA approaches for RFID uses CSMA MAC protocol of wireless networks and still suffer from traditional backoff scheme. In the existing protocols, how to set the backoff algorithm during channel access in order to make them efficient, however, is far less investigated. Most existing CSMA-based protocols use an arbitrary value without any discussion about its impact on the performance. Previous studies in MANETs [3], [9], [12] have outlined that the use of traditional binary exponential backoff (BEB) algorithm of CSMA protocols makes the channel access protocol built upon this approach to perform poorly. Based upon observed performance degradation, they proposed a novel adaptive backoff algorithm to overcome the poor performance of the traditional CSMA backoff. In what follows, we will adapt these protocols for broadcast communication because anti-collision scheme can not be directly applied in broadcast communication.

In this work, we investigate the characteristics in terms of throughput, fairness, latency and collision minimizing behavior of an adaptation of these proposed solutions in addition to SIFT protocol, proposed by Tay et al [13] in order to minimize the latency of reporting the event detected in Wireless Sensor Networks in the collision-free fashion, in large-scale RFID 
system. Our objective is to use these protocols to perform channel reservation through broadcasting message with almost zero-collision or in collision-free fashion. Once the channel is reserved by reader, it can communicate with tags located in its interrogation area in collision-free fashion. Since the communication between reader and tag is performed in reserved channel, each reader can maximize its reading throughput in a fair manner. Furthermore, our aim is, based on observed characteristics, to propose a novel medium access control protocol which could significantly improve the performance in terms of maximum throughput, fair channel sharing and minimum latency of RFID systems in large-scale deployment.

The rest of this paper is organized as follows. Section II reviews related works. Section III formally defines the problem, our objective, introduces the system model and the evaluated protocols. In Section IV, we evaluate their performance through simulation under various scenarios. Finally, Section V concludes by discussing future research direction.

\section{RELATED WORK}

In the following, we first overview the anti-collision protocols proposed in the literature of RFID systems. Thereafter, we introduce some adaptive CSMA protocols that are proposed to overcome the binary exponential backoff (BEB) algorithm.

\section{A. Reader-to-Reader Anti-collision}

PULSE [4] is CSMA-based protocol which is proposed to resolve hidden terminal issue. PULSE proposes the use of two non-interfering channels: control channel and data channel. It consists of periodically sending beaconing on control channel while reader is communicating with the tags on the data channel. In such a way, the beaconing transmission prevents any another reader to access to the channel. This leads to an increase of latency because the reader may need to wait thrice the beaconing transmission before any other channel access attempt. Moreover, its effectiveness in terms of collision avoidance comes at cost of high overhead of periodic beacon, which drastically impacts the energy-efficiency behavior when energy is a constraint. Furthermore, the channel access backoff parameter is set without any investigation.

Similar to PULSE protocol, DICA [10] is a distributed CSMA-based protocol with two channels, which aims to provide an energy-efficient collision avoidance protocol. During the channel access process, the reader may not need neither to periodically send beacon message nor to wait additional delay. To completely resolve hidden terminal and exposed terminal problems, DiCA adjusts the control channel communication range by doubling the radius from the data channel communication range. As in PULSE protocol, the backoff algorithm is designed without any investigation inducing misreading tags during the reader-to-tags communication.

Distributed Color Selection (DCS) [14] is one of the first distributed TDMA-based reader-to-reader anti-collision protocols. In DCS, communication is organized in groups of color timeslots, that are called frames or rounds. Each timeslot is composed of two phases: kick and transmission. The kick phase is used by readers that collided in the previous round in order to prevent a new collision in the current frame, while the latter phase is used by reader to read tags located in its interrogation range. Basically, It performs as follows. Each reader randomly selects a color timeslot where it can send the request to read tags during its transmission phase. If a collision is observed by colliding readers, they randomly select a new color and reserve them for the next frame. In the next frame, during the kick phase, colliding readers broadcast their color to all their neighborhood in order to prevent a new collision. Based upon information received from collide readers, neighborhood readers adjust their color so that a new collision could be avoided. Unfortunately DCS experiences a performance degradation due to the use of fixe number of colors. Because in a dense network, when the number of colors is too low, DCS experiences many collisions, while, in a low dense, when the number of used colors is too large, the throughput is degraded.To overcome this inflexibility, authors proposed Variable-Maximum DCS which dynamically adjusts the maximum number of colors regarding the percentage of successful transmissions monitored. A more efficient but centralized version has been proposed in [8].

Unlike CSMA-based protocols, these protocols necessitate a high level of synchronization among the backbone network formed by readers or an additional resource such as centralized server which can address the synchronization problem through wired or wireless connection. Moreover, they implicitly assume that when a collision occurs, colliding readers can detect the collision. In addition to the high level of synchronization requirement, in TDMA-based protocol, the use of dedicated server for explicit coordination restricts the use of these solutions into a limited environment.

\section{B. Adaptive CSMA-based protocols improvement}

In this section, we list the research works that propose various optimizations of the carrier sense multiple access with collision avoidance methods in order to improve the throughput or both the throughput and the fair channel allocation. The basic multiple access collision avoidance algorithm is based on the binary exponential backoff (BEB) algorithm to resolve collision problems in addition to the use of RTS/CTS mechanism which is firstly introduced by Karn [11] in order to overcome hidden terminal problem. MACAW [2] is one of the first approaches which have investigated the performance of the BEB algorithm in order to improve MACA [11] with the goal to maximize the throughput and the fairness property. It proposes to use the RTS-CTS-DS(Data Sending) mechanism prior to each data transmission with modified backoff algorithm, which is based on Multiplicative Increase and Linear Decrease(MILD) principle. In order to alleviate the unfairness problem, authors also propose a backoff counter copy scheme, which spreads the backoff counter to all contending stations through packet header.

Asymptotically Optimal Backoff (AOB) [5] is an improvement to the IEEE 802.11 protocol aiming to dynamically tune the backoff window size to achieve the theoretical capacity 
limit of the IEEE 802.11 protocol by estimating the slots utilization parameter and the average size of transmitted frames.

Optimal constant backoff (OCB) [1] is an enhancement of IEEE 802.11 backoff algorithm and aims to increase both the throughput and fairness. Bianchi et al [3] showed that the network throughput can be maximized if the backoff counter is set to be proportionnal to the number of active terminals.

\section{PRELIMINARIES}

In this section, we first give an overview of our system model. Then, we introduce our objective and the performance metrics. Finally, we detail the studied protocols.

\section{A. System Model}

We consider a large-scale RFID system with multiple readers and homogeneous local density of RFID tags within the interrogation area. The readers are randomly deployed with uniform distribution on 2D plane. Readers are assumed to have homogeneous property. Therefore, their communication range is assumed to be the same. Similar to the assumption used in [10], in this paper, we assume that there are two communication channels: control channel and data channel. We assume the existing of an overlapping area in their interrogation areas. Note that the knowledge of these overlapping areas is not a necessite in this work. We assume that readers are able to accurracy estimate their neighborhood reader size in the case of adaptive backoff schemes that are based on the number of active readers. As this assumption is not acceptable in real RFID systems deployment due to the wireless channel behavior and the fact that the ideal estimation mechanism does not exist, in Section IV, we introduce some errors in the estimation in order to investigate the sensitivity of these protocols to these possible errors.

\section{B. Problem Statement}

Regardless to the type of RFID tags used within RFID system and their hardware requirements, the focus of this work is: How to efficiently reserve channel in large-scale RFID readers network in order to guarantee reader-to-tags communication without any interfering reader by inspiring from MANETs CSMA techniques.

We assume that readers have two wireless interfaces. The first one is the classical tag reading interface and the second one is a adhoc wireless communications allowing a reader backbone network. In our approaches, there are two ways to apply MANETs CSMA techniques to reduce collisions in RFID networks. In both approaches, there are two to consider: reader-to-reader network communication or backbone network problem $^{1}$ and reader-to-tags communication problem. The first one is a 2-step approach in which readers first send a channel reservation request on the adhoc medium in a CSMA fashion. Once the request does not collide, they can read tags in their vicinity (so activate the second interface). This ensures that no collisions occur during the second step. The second one is to activate both interfaces similarly and detect collisions thanks to

\footnotetext{
${ }^{1}$ We assume that readers have a $\mathrm{P} 2 \mathrm{P}$ wireless communication mean.
}

the adhoc interface. The idea of this paper is this to investigate how efficient are some well-known CSMA-based protocols, that have proved their efficiency in MANETs or VANETs, in order to maximize the RFID application requirement such as throughput and the fairness property with regards to these approaches. The latter property guarantees that there is no reader starvation problem. For the sake of clarity, in this paper, we will mainly focus on the first approach and leave for future works the investigation of results of this current approach to propose an efficient mechanism for the second approach.

\section{Performance metric}

In the section, we will evaluate the investigated protocols based on these performance metrics:

- Collision: We can distinguish two types of collisions: channel access collision and reading collision. When the former occurs, we consider that the request to read tags which follows the channel reservation message to be lost while the latter problem will just impact the throughput of tags reading. Note that due to some of our assumptions, in this work, the latter can not happen.

- Throughput: We define the throughput as the average number of successful query sections per reader.

- Jain's Fairness Index: In the literature, the fairness property is generally evaluated with the Jain's fairness index, which describes how similar and fair the resources allocated to each reader are. It is computed as follows:

$$
J=\frac{\left|\sum_{i=1}^{N} X_{i}\right|^{2}}{N \sum_{i=1}^{N} X_{i}^{2}}
$$

where $\mathrm{N}$ and $X_{i}$ are respectively the number of readers in the network and the throughput of the $i$-th reader.

- Latency: It represents the time span between the request generation and the successful transmission.

\section{Protocols overview}

\section{Neighbor-based Adaptive Contention Window}

Bianchi et al in [3] showed that to achieve the optimal network throughput, the minimum contention $C W_{\min }$ of backoff algorithm must be stated according to the number of contending stations. They set the backoff counter as $W=\bar{\Gamma} \sqrt{2 T}$ where $\bar{\Gamma}$ is the estimated number of contending stations, obtained through channel activity monitoring, and $T$ is the data transmission time (i.e., the sum of data and ACK transmissions). As the reservation request transmission is done in a broadcast manner, we will consider only the reservation message transmission. Based on our assumption on the neighborhood size knowledge, we set $\bar{\Gamma}$ to be equal to the neighborhood size.

\section{Maximum Backoff or Reverse-Backoff}

Generally speaking, the probability of a collision decreases when the contention window size increases. Based on this, [12] introduces reverse backoff (RB). RB starts with a backoff with maximum value decreased every time a beacon expires 
until some maximum backoff counter, which does not induce the beaconing expiration. It copes with collision problem during the channel access because the backoff counter is large. However, the achieved value depends on the beacon lifetime. As our goal is to minimize the channel access collision and that message expiration is not part of this work, in the following, we set the backoff counter to be equal to the maximum value of IEEE 802.11 protocol $(C W=1023)$.

\section{Idle Sense}

Idle Sense [9] aims at maximizing the throughput and providing a short-time fairness for IEEE 802.11 DCF. Instead of performing the binary exponential backoff algorithm of IEEE 802.11 after a collision or failed transmission due to channel behavior, Idle Sense dynamically adjusts the contention window size so that the contention window size of all nodes converges in a full distributed way to similar values. It performs as follows: each host estimates $n_{i}$, the number of consecutive idle slots between two transmission attempts. According to the observed values of $n_{i}$, every maxtrans transmissions, it computes $\hat{n}_{i}$, which represents the average. Then, it uses the average value to adjust its contention window size to target value $n^{\text {target }}$ computed numerically based on the IEEE 802.11 PHY and MAC layers parameters such as the slot size and average collision size. Idle Sense is based on Additive Increase Multiplicative Decrease (IAMD) mechanism to adjust the window. The heuristic is performed as follows :

- if $\hat{n}_{i} \geq n^{\text {target }} C W \longleftarrow \alpha . C W$

- if $\hat{n}_{i}<n^{\text {target }} C W \longleftarrow C W+\epsilon$

where $\alpha$ and $\epsilon$ are parameters of the AIMD algorithm. While in the first version of Idle Sense [9], maxtrans was chosen by simulation and fixed, in [7] they proposed adaptive algorithm to update the value of maxtrans in order to speed up the convergence of the average $\hat{n}_{i}$ to the target value. Idle Sense is designed for unicast data transmission, because nodes may need to detect a collision in order to adjust their contention window, while in our work, the communication is performed through broadcast transmission. As we assume that each reader can track its right neighborhood size and according to the value of slot size selected in this work and formulas (4)-(5) and (10)(12) of [9], we provide in Table I for each neighborhood size its optimal contention window for the target value of 9.41 .

TABLE I: Values of CW for $n_{i}^{\text {target }}=9.41$

\begin{tabular}{|c|c|}
\hline$\Gamma$ & $\mathrm{CW}$ \\
\hline \hline 2 & 39.61 \\
4 & 79.23 \\
6 & 118.84 \\
8 & 158.46 \\
10 & 198.07 \\
\hline
\end{tabular}

\begin{tabular}{|c|c|}
\hline$\Gamma$ & CW \\
\hline \hline 12 & 237.69 \\
14 & 277.31 \\
16 & 316.92 \\
18 & 356.54 \\
20 & 396.15 \\
\hline
\end{tabular}

\begin{tabular}{|c|c|}
\hline$\Gamma$ & $\mathrm{CW}$ \\
\hline \hline 22 & 435.77 \\
24 & 475.38 \\
26 & 515.00 \\
28 & 554.62 \\
30 & 594.23 \\
\hline
\end{tabular}

\section{Sift}

SIFT [13] is motivated by the limitation of classical CSMAbased approach in WSN when several nodes with spatiallycorrelated data simultaneously attempt to report data to the sink node. It aims at reducing the latency and the collisions with a competitive throughput by using a suppression mechanism at MAC Layer. Instead all nodes report data, only few nodes called the first $R$ nodes report their data in a collisionfree fashion. Instead of using BEB algorithm to access the channel, it uses a fixed-size contention window with a nonuniform geometrically-increasing probability distribution for picking a transmission slot in the window. This avoids the shortcoming due to the adaptive increasing of window size when collision occurs. This distribution function is given as:

$$
p_{r}=\frac{(1-\beta) \beta^{C W}}{1-\beta^{C W}} \cdot \beta^{-r}, \text { for } \mathrm{r}=1 \ldots . . \mathrm{CW}
$$

where $0<\beta<1$ is a distribution parameter. This distribution function $p_{r}$ increases exponentially with $r$. All contending nodes select a new random contention slots and repeat the same process. Although SIFT is proposed with the aim of reducing the number of collisions through the suppression mechanism, collisions do still exist. Our objective is to use SIFT in order to allow collision-free channel access for all contending nodes.

\section{PERformance Evaluation}

We implemented these listed MAC protocols using WSNet 2 in order to fairly evaluate their performance under various networks scenarios. Table II sums up all parameters. The simulation scenario is a $100 \mathrm{~m} \times 100 \mathrm{~m}$ rectangle in which 50 to 300 nodes are randomly deployed. Each simulation lasts 500s.

TABLE II: Simulation models parameters

\begin{tabular}{|c|c|c|c|}
\hline Parameters & Value & & \\
\hline Radio bit rate & $64 \mathrm{Kbps}$ & Parameters & Value \\
\hline Transmission power & $0 \mathrm{dBm}$ & Query period & $500 \mathrm{~ms}$ \\
\hline Reception Sensitivity & $-92 \mathrm{dBm}$ & Packet size & 34 Bytes \\
\hline Radio range & $20 \mathrm{~m}$ & Reading Period & $600 \mathrm{~ms}$ \\
\hline$T_{\text {slot }}$ & $15 \mu \mathrm{s}$ & error & $5-20(\%)$ \\
\hline Simulation time & $500 \mathrm{~s}$ & $C W_{S I F T}$ & 32 \\
\hline
\end{tabular}

\section{A. Impact of node density}

In this part, we evaluate the performance of these listed MAC protocols according to the number of deployed readers while the number of tags are fixed.

\section{Throughput}

Fig. 1 illustrates the system throughput according to the number of readers. Results show that all protocols exhibit similar performance when the number of readers increases. This decreasing behavior is mainly due to the increasing number of contending readers with a constant simulation duration. Thus, the number of sections for each reader also decreases. Results also show that whatever the number of readers, Idle Sense, Neighbor-based and maximum backoff outperform SIFT. SIFT does not use suppression mechanism in our study, so it can not completely mitigate the collisions.

\footnotetext{
${ }^{2}$ Wsnet:http://wsnet.gforge.inria.fr/
} 


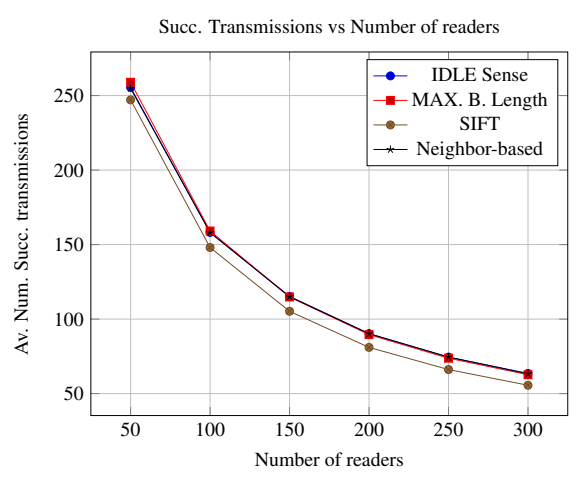

Fig. 1: Throughput per reader vs Number of readers.

\section{Number of Collisions}

Fig. 2 shows that whatever the number of readers, the maximum backoff is the solution that better mitigate collisions thanks to a large window size which minimizes the probability of collision. It is followed by the protocols that use the neighborhood size while SIFT performs poorly.

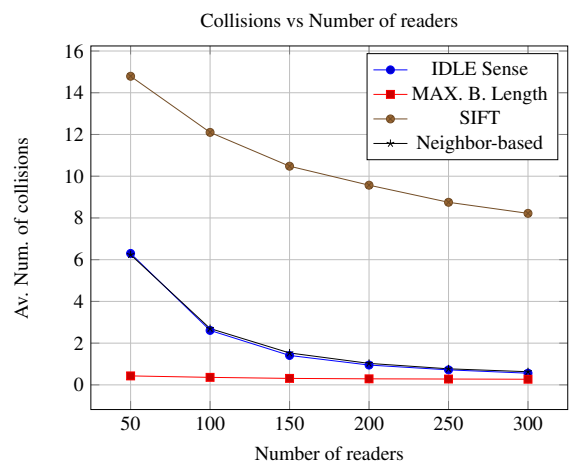

Fig. 2: Average number of collisions vs Number of readers.

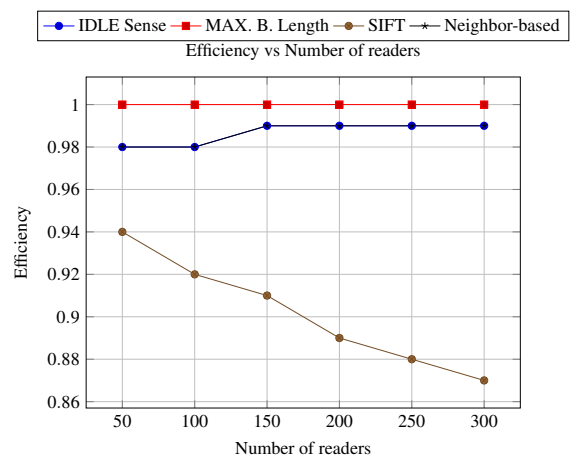

Fig. 3: Protocols efficiency vs Number of readers.

\section{Jain's Fairness Index}

Fig. 4 shows that as the number of readers increases, the Jain's fairness indexes of Idle Sense and neighbor-based protocols decrease to reach the value of $40 \%$ with maximum backoff protocol and 35\% for two other protocols. While SIFT keeps its fairness index around $60-50 \%$ as the number of reader increases. SIFT is thus fairer than the maximum backoff and the neighborhood size knowledge based approach respectively. This also means that their use in RFID systems, where the reader may need to keep the channel in order to read tags, after its successful channel reservation transmission is not benefit for these approaches, while their design goal provides a fair channel sharing. So we may need another approach which can use a priority according to their number of attempts.

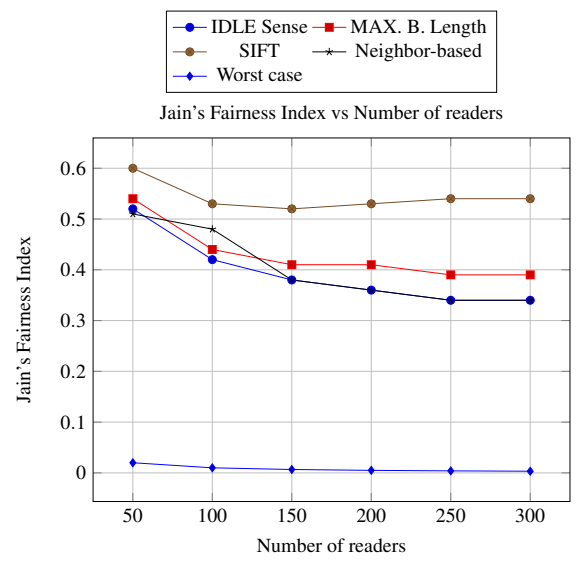

Fig. 4: Jain's Fairness Index vs Number of readers.

\section{Latency}

Fig. 5 shows the delay which the query spans in average before its transmission. Results show that protocols behave similarly as the number of reader increases. Neighborhood size knowledge based approach outperforms the maximum backoff protocol and SIFT protocol respectively. The poor performance of SIFT is due to the fact that after a successful or collision transmission, all contending readers may need to renew the random contention slot selection process. So, its latency increases with the number of readers in the system.

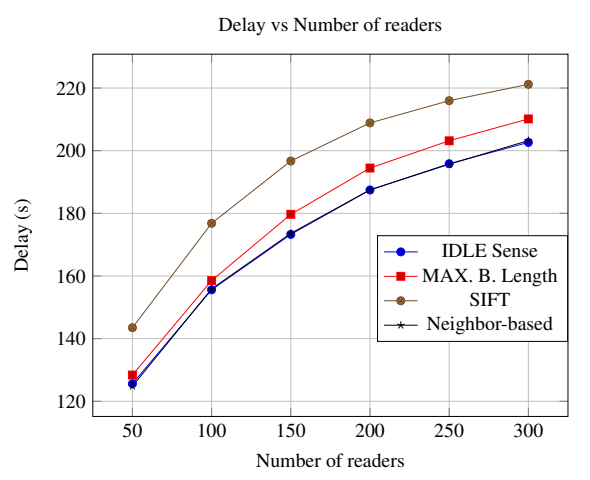

Fig. 5: The average latency vs Number of readers

\section{B. Impact of error}

Previously, we have considered that each reader can tracks its right neighborhood size. Now, in order to show the impact 
of error which can occur in the estimation of the neighborhood size, we introduce errors in the proper measure of the neighborhood size. We present only the throughput performance for Idle Sense and Neighbor-based protocols because they use the neighborhood size knowledge to estimate their contention window size.

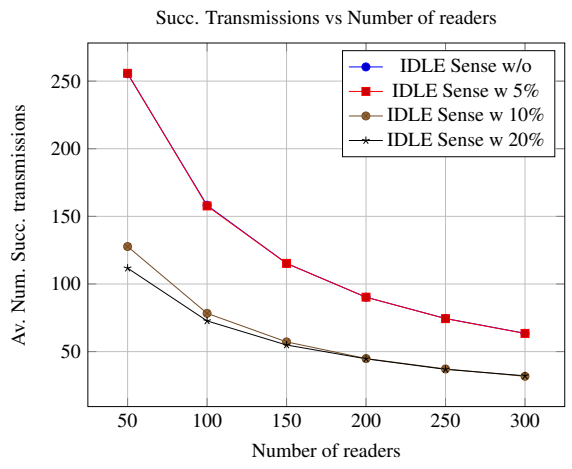

Fig. 6: Idle Sense: The impact of error vs Number of nodes

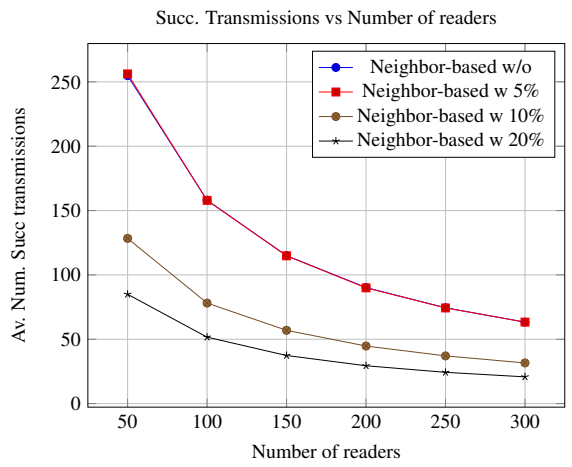

Fig. 7: Neighbor-based: Impact of error vs Number of nodes

Fig.6 and 7 show the average throughput per reader when the number of nodes increases. For both approaches, when the error due to the estimation of the neighborhood size is less than or equal to $5 \%$, the performance does not change compared to the best case with in error-free estimation. While, by increasing the error value by 5 at each step from $5 \%$ to $20 \%$, we show that both approaches perform poorly. Performance degradation is more important for Neighbor-based than for Idle Sense because in the latter protocol, as the number of readers increases, both $15 \%$ and $20 \%$ exhibit a similar performance. These results show that both approaches are sensitive to the error in the neighborhood size estimation when the error is higher than 5\%. Therefore, in our future work, if these approaches are to be used, we should try to guarantee an effectiveness estimation algorithm so that the error introduced in our measures is lower than $5 \%$.

\section{Discussion AND CONCLUSIONS}

The study presented in this paper allows the highlighting of the different advantages and drawbacks brought by the use of MANETs CSMA approaches in a 2-step tag reading approach.
It allows the selection of the most appropriated one with regards to application requirements. For instance, it shows that for an application with low delay, low energy consumption but accurate reading requirements, MAX B length proposed the best trade-off. If the most important criteria for the application is to give equal access time to readers, SIFT should be chosen. None of the MANETs CSMA approaches improves all criteria at the same time. Based on these results, our future works will consist in proposing an efficient reader-to-reader anticollision scheme with regards to the maximum of criteria by combining CSMA algorithm strength. We will lead these analysis by considering that both wireless interfaces of readers are activated similarly. In light of this study's results, a IDLE sense fashion approach combined with SIFT may provide the best tradeoff but further investigation is needed since expected results are not so straight forward since when a collision occurs at tags, tags not lying in the field overlapping areas will still be read.

\section{ACKNOWLEDGMENTS}

This work is partially supported by a grant from CPER Nord-Pas-de-Calais/FEDER Campus Intelligence Ambiante.

\section{REFERENCES}

[1] H. Anouar and C. Bonnet. Optimal constant-window backoff scheme for IEEE 802.11 DCF in single-hop wireless networks under finite load conditions. In Journal of Wireless personal com., Vol.43:(4), pp.15831602, 2007.

[2] V. Bharghavan, A. Demers, S. Shenker, L. Zhang. MACAW: A Media Access Protocol for Wireless LAN's, In Proc. ACM SIGCOMM, 1994.

[3] G. Bianchi, L. Fratta, M. Oliveri. Performance Evaluation and Enhancement of the CSMA/CA MAC Protocol for 802.11 Wireless LANs, In Proc. PIMRC, 1996.

[4] S. Birari, S. Iyer. PULSE: a MAC protocol for RFID networks, In Proc. EUC Workshops, 2005.

[5] L. Bononi, M. Conti, and E. Gregori. Runtime Optimization of IEEE 802.11 Wireless LANs Performance. In IEEE Transactions on Parallel and Distributed Systems, Vol.15:(1), pp.66-80, 2004.

[6] K. Finkenzeller. RFID Handbook: Fundamentals and Applications in Contactless Smart Cards, Radio Frequency Identification and Near-field Communication, Third Edition. John Wiley \& Sons, 2010.

[7] Y. Grunenberger, M. Heusse, F. Rousseau, A. Duda. Experience with an implementation of the Idle Sense wireless access method, In Proc. ACM CoNEXT, 2007.

[8] E. Hamouda, N. Mitton and D. Simplot-Ryl. Reader Anti-Collision in Dense RFID Networks With Mobile Tags, In IEEE Intern. RFID-TA, 2011.

[9] M. Heusse, F. Rousseau, R. Guillier, A. Duda. Idle sense: an optimal access method for high throughput and fairness in rate diverse wireless LANs, In Proc. ACM SIGCOMM, 2005.

[10] K. Hwang, K. Kyung-Tae, E. Doo-Seop. DiCa: Distributed Tag Access with Collision-Avoidance Among Mobile RFID Readers, In Proc. EUC Workshops, 2006.

[11] P.Karn. MACA: A new Channel Access Method for Packet Radio. In Proc. of ARRL/CRRL Amateur Radio 9th Computer Networking Conference, 1990.

[12] R. Stanica, E. Chaput, A.-L. Beylot. Enhancements of IEEE 802.11p Protocol for Access Control on a VANET Control Channel, In Proc. ICC, 2011.

[13] K. Jamieson, H. Balakrishnan, and Y.C. Tay Sift: a MAC protocol for event-driven wireless sensor networks, In Proc. 3rd European Workshop on Wireless Sensor Networks, pp. 260-275, 2006.

[14] J. Waldrop, D.W. Engles, S.E. Sarma. Colorwave: An anticollision algorithm for the reader collision problems, in Proc. ICC, 2003. 